(C) 2013 IEEE. Personal use of this material is permitted. Permission from IEEE must be obtained for all other uses, in any current or future media, including reprinting/republishing this material for advertising or

promotional purposes, creating new collective works, for resale or redistribution to servers or lists, or reuse of any copyrighted component of this work in other works.

\title{
Delay Distribution Analysis of Wireless Personal Area Networks
}

\author{
Pangun Park ${ }^{1}$, Piergiuseppe Di Marco ${ }^{2}$, Carlo Fischione ${ }^{2}$ and Karl Henrik Johansson ${ }^{2}$
}

\begin{abstract}
Characterizing the network delay distribution is a fundamental step to properly compensate the delay of Networked Control Systems (NCSs). Due to the random backoff mechanism employed by Wireless Personal Area Network (WPAN) protocols, it is difficult to derive such a distribution. In this paper, the probability distribution of the delay for successfully received packets in WPANs is characterized. The analysis uses a moment generating function method based on an extended Markov chain model. The model considers the exponential backoff process with retry limits, acknowledgements, unsaturated traffic, and variable packet size, and gives an accurate explicit expression of the probability distribution of the network delay. The probability distribution of the delay is a function of the traffic load, number of nodes, and parameters of the communication protocol. Monte Carlo simulations validate the analysis for different network and protocol parameters. We show that the probability distribution of the delay is significantly different from existing network models used for NCS design. Furthermore, the parameters of the communication protocol result to be critical to stabilize control systems.
\end{abstract}

Keywords: Delay distribution, Wireless Personal Area Networks, Networked Control Systems, Markov chain.

\section{INTRODUCTION}

The wireless technology has a tremendous potential to improve the efficiency of control systems [1]. The Wireless Personal Area Networks (WPANs) have received considerable attention as major low data rate and low power standard for Wireless Sensor Network (WSN) applications, for instance, in building automation and process control [1], [2]. Networked Control Systems (NCSs) require practical delay models in order to properly design the control strategy. Otherwise, the network delay can degrade the performance of NCSs and even destabilize the system [3], [4]. The network delay models currently used for NCSs can be classified into two categories: constant delay and variable delay. Several works in literature investigate the NCS design by assuming the constant delay model [4]. Although this technique is useful for developing control laws of NCSs, it fails to assess general stability condition for random communication delays. The random delay significantly reduces the maximum allowable transfer interval to stabilize a NCS with respect to the constant delay model [5]. In particular, the delay jitter of a network is difficult to compensate in control loops, especially

\footnotetext{
${ }^{1}$ P. Park is with the Department of Electrical Engineering and Computer Sciences, University of California, Berkeley, CA 94709 USA. Email: \{pgpark\} deecs.berkeley.edu. P. Park was with the ACCESS Linnaeus Center, Electrical Engineering, Royal Institute of Technology, Stockholm, Sweden. when contributing to this work.

${ }^{2}$ P. Di Marco, C. Fischione, K. H. Johansson are with the ACCESS Linnaeus Center, Electrical Engineering, Royal Institute of Technology, Stockholm, Sweden. E-mails: $\{$ pidm, carlofi, kallej\}@ee.kth. se. The work of the authors was supported by the EU projects Hycon2 and Hydrobionets and the Swedish Research Council.
}

if the delay variability is large [6]. Many protocols for wireless communications [2], [7], introduce random delays that can vary significantly from packet to packet. One of the common approaches to compensate random delays is to define the finite set of possible network delays and compute the transition probability between the states. In this way, the NCS can be modeled as a jump linear Markov system where the random variation of network delays corresponds to randomly varying structure of the state-space model [8]. In [8], the transition probability matrix is assumed to be known. In [9], a simple Markov chain is used to model the network delay depending on the traffic load of a network. Each state of the Markov chain presents a particular delay distribution. However, the transition probabilities between the state and the delay distribution model of each state are not explicitly defined to be used in practice. In [10], a continuous probability density function (PDF) is used to model the random delay process in order to analyze the stability of the NCS. Most studies describe the random delay by an Independent and Identically Distributed (IID) random process with a known PDF. The PDF of the delay is known a priori in most methods. However, in practice, the network delay depends on the traffic load, number of nodes, and parameters of the communication protocol, which typically vary over time.

This paper focuses on the IEEE 802.15.4 WPAN protocol, because it is becoming the most popular standard for low data rate and low power WSNs in many application domains. A simulations-based study of the delay of WPANs has been proposed [11] without paying attention to delay jitter. Several analytical models have been developed by using the Markov chain model of Bianchi [12] for Wireless Local Area Networks (WLANs) [7]. The probability distribution of the delay for WLANs is investigated in [13]. A Markov model for WPANs based on Bianchi's model has been proposed in [14]. However, the packet retransmission and the delay distribution are not considered. Our previous work in [15] introduces a generalized Markov chain model for the Carrier Sense Multiple Access with Collision Avoidance (CSMA/CA) mechanism of WPANs [2], but only average performance indicators are derived.

To the best of our knowledge, there is no explicit derivation of the delay distribution of WPANs. We use a moment generating function method based on an extended Markov chain model to derive the practical probability distribution of the delay. We show that existing analytical studies are not adequate to capture the real-world protocol behavior of the delay distribution. We consider simultaneously the maximum number of packet transmissions attempts, the 


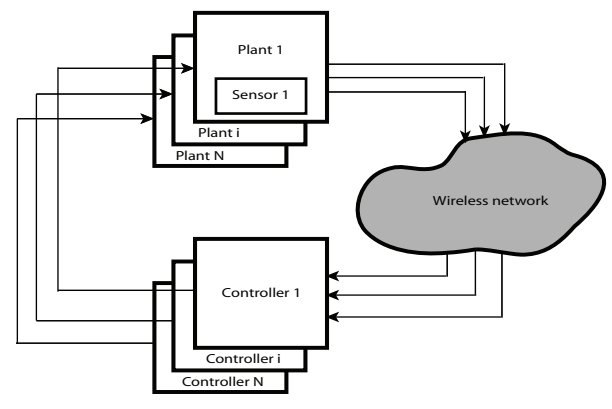

Fig. 1. Overview of the NCS setup. $N$ plants need to be controlled by $N$ controllers. The wireless network closes the loop from the sensor nodes to the controllers.

acknowledgement mechanism, unsaturated traffic load, and packet size. These characteristics make the delay analysis of WPANs proposed in this paper different from the previous results available from the literature.

Several parameters including the traffic load, number of nodes, physical link quality, and network protocol parameters significantly affect the network performance in terms of reliability, delay, channel utility, and energy consumption. The performance of NCSs is improved by tuning those parameters, and several adaptive algorithms are developed and used in practice [16]. However, it is not always possible to tune those parameters depending on the particular scenario [16]. For example, changing the sampling period of the plant and the number of sensor nodes attached to the plant is not a trivial task in the physical environment. We illustrate the effect of communication protocol parameters on the performance of state feedback control systems.

The remainder of this paper is as follows. In Section II, we introduce the Markov chain model of CSMA/CA mechanism. Section III analyzes the distribution of the service time and delay for successfully received packet. In Section IV, we validate our analysis by Monte Carlo simulations. We also show how different network parameters affect the performance of a control system. Section V concludes the paper.

\section{Markov Chain Model}

The problem considered is depicted in Fig. 1, where multiple plants are controlled over a WPAN. $N$ plants contend to transmit sensor measurements to the controller over a wireless network that induces time varying delays. We assume that the controller commands are always successfully received by the actuator [1]. We consider a single cell wireless network, where every node can hear the other nodes of the network. We assume that a sensor node is attached to each plant. The CSMA/CA mechanism of WPANs is used to determine which sensor node accesses the wireless channel. The wireless channel is assumed to be perfect and the collision may occur only when two (or more) frames are transmitted at the same time. Throughout this paper we consider control applications where nodes asynchronously generate packets with probability $1-q$, when a node sends a packet successfully, discard a packet or the sampling interval is expired. Otherwise a node stays for $L_{0} S_{b}$ seconds without

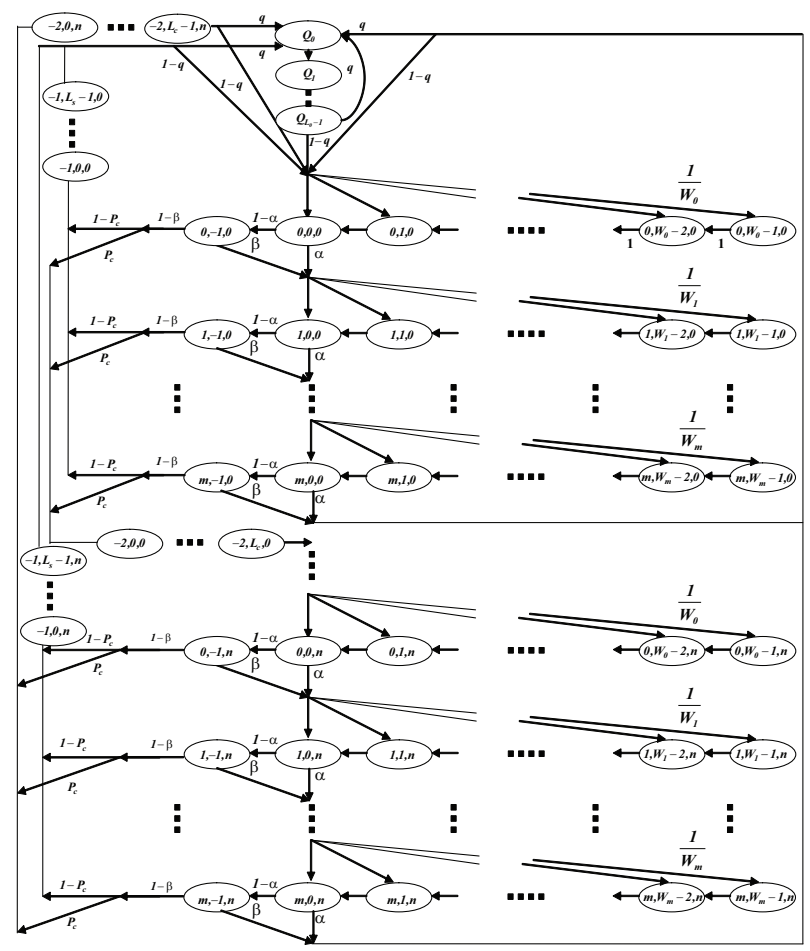

Fig. 2. Markov chain model proposed in [15] for the CSMA/CA mechanism of WPANs. Note that $L_{s}$ is the successful transmission time and $L_{c}$ is the collision time.

generating packets with probability $q$, where $L_{0}$ is an integer and $S_{b}$ is the time unit aUnitBackoffPeriod corresponding to 10 bytes. The data packet transmission is successful if an acknowledgement packet is received.

An accurate analytical model of the CSMA/CA mechanism was presented in [15], where we introduced a Markov chain model for CSMA/CA mechanism. We build upon this model to analyze the delay distribution in Section III.

Let $s(t), c(t)$ and $r(t)$ be the stochastic process representing the backoff stage, the state of the backoff counter and the state of retransmission counter at time $t$, respectively, experienced by a node to transmit a packet, as indicated in Fig. 2. By assuming independent probability that nodes start sensing, the stationary probability $\tau$ that the node attempts a first carrier sensing in a randomly chosen slot time is constant and independent of other nodes. It follows that $(s, c, r)$ results in a three dimensional Markov chain. The protocol parameters are denoted by $W_{0}=2^{\operatorname{macMinBE}}, m_{0}=\operatorname{macMinBE}, m_{b}=\operatorname{macMaxBE}, m=$ macMaxCSMABackoffs, $n=$ macMaxFrameRetries. The states from $\left(i, W_{m}-1, j\right)$ to $\left(i, W_{0}-1, j\right)$ represent the backoff states. States $\left(Q_{0}, \ldots, Q_{L_{0}-1}\right)$ consider the idle state that the packet queue is empty and node is waiting for new packet arrival. Note that the idle states $\left(Q_{0}, \ldots, Q_{L_{0}-1}\right)$ take into account the unsaturated traffic condition. States $(i, 0, j)$ and $(i,-1, j)$ represent the first clear channel assessment $\left(\mathrm{CCA}_{1}\right)$ and second assessment $\left(\mathrm{CCA}_{2}\right)$, respectively. $\alpha$ is the probability that $\mathrm{CCA}_{1}$ is busy, and $\beta$ is the probability that $\mathrm{CCA}_{2}$ is busy. If all nodes transmit with probability $\tau$, 
the collision probability $P_{c}$ is

$$
P_{c}=1-(1-\tau)^{N-1}
$$

where $N$ is the number of nodes. The term $P_{c}$ is the probability that at least one of the $N-1$ remaining nodes transmit in the same time slot. States $(-1, k, j)$ and $(-2, k, j)$ consider the successful transmission and packet collision. The probability $\tau$ that a node attempts $\mathrm{CCA}_{1}$ and the busy probabilities $\alpha$ and $\beta$ are derived by solving the state transition probabilities associated with the Markov chain model [15]. In particular, we use the solutions of $\tau, \alpha$ and $\beta$ to derive the probability distribution function of the service time and of the delay to transmit a packet successfully.

\section{Delay Analysis of CSMA/CA MeChanism}

In this section we give one of the core contribution of the paper, which is the derivation of the distribution of the service time $T_{s}$ and the distribution of the delay $T_{d}$ experienced by a packet to be successfully received. These distributions are discrete because the time unit of the backoff timer is an integer given by aUnitBackoffPeriod.

We derive the distribution of $T_{d}$ by its probability generating function (PGF) $D(Z)$. Recall that the moments of $T_{d}$ of any order and degree can be obtained by derivation of $D(Z)$. For example, the mean and variance of $T_{d}$ are

$$
\begin{aligned}
\mathbb{E}\left[T_{d}\right] & =D^{\prime}(1) \\
\mathbb{E}\left[\left(T_{d}-\mathbb{E}\left[T_{d}\right]\right)^{2}\right] & =D^{\prime \prime}(1)+D^{\prime}(1)-\left\{D^{\prime}(1)\right\}^{2},
\end{aligned}
$$

where ' indicates the derivative with respect to $Z$. Same argument applies for the derivation of the distribution of $T_{s}$ by its PGF $B(Z)$. The expression of $B(Z)$ is given by Proposition 1 , and $D(z)$ is provided by Proposition 2 later in this section. Two intermediate technical results are as follows: (a) the derivation of PGF of the random backoff process, (b) the derivation of the transfer function of sensing fails due to the busy channel. Details follow in the sequel.

The PGF of the random backoff process $H_{i}(Z)$ is given by the product from the 0 to the $i$-th stage of Fig. 2 as

$$
H_{i}(Z)=\prod_{k=0}^{i} W_{k}(Z)
$$

where $W_{i}(Z)$ is the PGF of the backoff time at the $i$-th:

$W_{i}(Z)= \begin{cases}\sum_{l=0}^{2^{i} W_{0}-1} \frac{M_{d}(Z)^{l}}{2^{i} W_{0}}=\frac{1-Z^{2^{i} W_{0}}}{2^{i} W_{0}(1-Z)} & \text { if } i \leq m_{b}-m_{0} \\ \sum_{l=0}^{2^{m_{b}}-1} \frac{M_{d}(Z)^{l}}{2^{m_{b}}}=\frac{1-Z^{2^{m_{b}}}}{2^{m_{b}}(1-Z)} & \text { otherwise }\end{cases}$

where we used that $M_{d}(Z)=Z$, because the decrement of backoff counter happens with probability 1 . Then, we have the following result:

Lemma 1: Let $C_{\alpha \beta}(i)$ be the set containing all the combinations of choosing $i$ elements out of a set of busy channel probabilities $\{\alpha,(1-\alpha) \beta\}$. Let $S_{c}(Z)$ be the PGF of the sensing time. Then the transfer function of the event of $i$ sensing fails due to the busy channel condition is

$$
G_{i}(Z)=\sum_{k=1}^{2^{i}} C_{\alpha \beta}^{k}(i) S_{c}(Z)^{N_{\alpha}^{k}(i)+2 N_{\hat{\beta}}^{k}(i)}
$$

where $S_{c}(Z)=Z$ is the sensing time, $C_{\alpha \beta}^{k}(i)$ returns the k-th combination of $C_{\alpha \beta}(i)$ and $N_{\alpha}^{k}(i), N_{\beta}^{k}(i)$ returns the number of $\alpha$ and $(1-\alpha) \beta$ in the combination, respectively.

Proof: During the backoff process, the backoff timer at $i$-th stage decreases by one unit time regardless of the channel state ${ }^{1}$. When the random backoff time of node is equal to zero the node goes to sensing state. If the medium is idle (with probability $1-\alpha$ ) at $\mathrm{CCA}_{1}$ then the node goes to $\mathrm{CCA}_{2}$. If an ongoing transmission (with probability $\alpha$ ) is detected, the backoff exponent is increased by one and a random backoff time is generated until the maximum number of stages is reached. The node repeats the same mechanism during the second sensing in Fig. 2. The total number of combinations for $i$ elements is equal to $2^{i}$ and are collected in the set $C_{\alpha \beta}(i) . C_{\alpha \beta}^{k}(i)$ is one of the combinations out of $2^{i}$ events. The function $2 N_{\beta}^{k}(i)$ gives the sensing fails at second sensing state from which Eq. (4) follows.

Now, we derive the PGF of $T_{s}$ and $T_{d}$ :

Proposition 1: Let $B(Z)$ be the PGF of the service time $T_{s}$. Then,

$$
\begin{aligned}
B(Z)= & S_{t}(Z) \sum_{j=0}^{n} \operatorname{Pr}\left(\mathcal{S}_{j} \mid \mathcal{U}_{t}\right) C_{t}(Z)^{j}\left(S_{c}(Z)^{2}\right. \\
& \left.\times \sum_{i=0}^{m} G_{i}(Z) H_{i}(Z)\right)^{j+1}+\sum_{j=0}^{n} \operatorname{Pr}\left(\mathcal{C}_{j} \mid \mathcal{U}_{t}\right) \\
& \times\left(C_{t}(Z) S_{c}(Z)^{2} \sum_{i=0}^{m} G_{i}(Z) H_{i}(Z)\right)^{j} G_{m+1}(Z) \\
& \times H_{m+1}(Z)+\operatorname{Pr}\left(\mathcal{F} \mid \mathcal{U}_{t}\right)\left(C_{t}(Z)\right. \\
& \left.\times S_{c}(Z)^{2} \sum_{i=0}^{m} G_{i}(Z) H_{i}(Z)\right)^{n+1}
\end{aligned}
$$

where

$$
\begin{aligned}
& \operatorname{Pr}\left(\mathcal{S}_{j} \mid \mathcal{U}_{t}\right)=\frac{P_{c}^{j}\left(1-P_{c}\right)\left(1-x^{m+1}\right)^{j+1}}{\operatorname{Pr}\left(\mathcal{U}_{t}\right)}, \\
& \operatorname{Pr}\left(\mathcal{C}_{j} \mid \mathcal{U}_{t}\right)=\frac{P_{c}^{j}\left(1-x^{m+1}\right)^{j} x^{m+1}}{\operatorname{Pr}\left(\mathcal{U}_{t}\right)} \\
& \operatorname{Pr}\left(\mathcal{F} \mid \mathcal{U}_{t}\right)=\frac{P_{c}^{n+1}\left(1-x^{m+1}\right)^{n+1}}{\operatorname{Pr}\left(\mathcal{U}_{t}\right)}
\end{aligned}
$$

with,

$$
\begin{aligned}
\operatorname{Pr}\left(\mathcal{U}_{t}\right)= & \frac{1-\left(P_{c}\left(1-x^{m+1}\right)\right)^{n+1}}{1-P_{c}\left(1-x^{m+1}\right)}\left(1-P_{c}+P_{c} x^{m+1}\right) \\
& +P_{c}^{n+1}\left(1-x^{m+1}\right)^{n+1}
\end{aligned}
$$

and $G_{i}(Z)$ is given by Lemma 1 .

Proof: The PGF of the service time is derived by considering the Markov chain given in Fig. 2. Whenever the node succeeds two CCAs, a transmission commences. According to the probability $P_{c}$ of a collision seen by a packet being transmitted on the medium, the node has a probability $1-P_{c}$ to finish the transmission after $S_{t}(Z)$,

\footnotetext{
${ }^{1}$ Note that this mechanism is different from WLAN which senses the channel state during the backoff time
} 


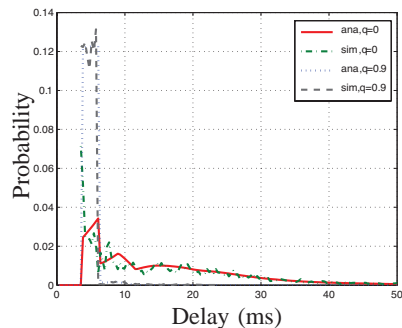

(a) $N=10, m_{b}=5$

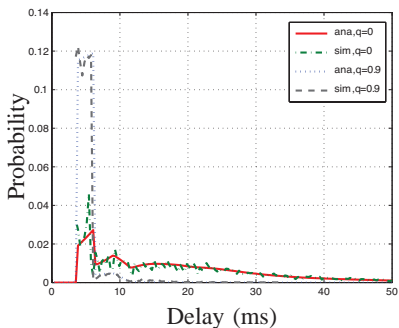

(b) $N=30, m_{b}=5$

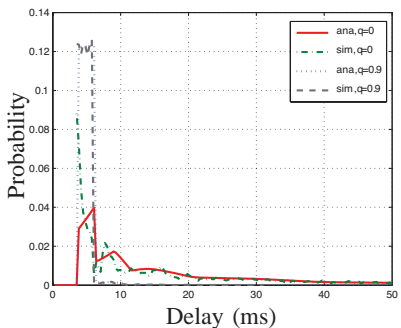

(c) $N=10, m_{b}=8$

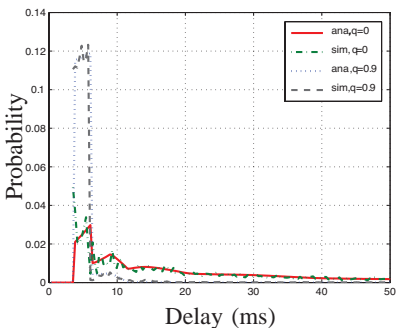

(d) $N=30, m_{b}=8$

Fig. 3. Probability distribution of delay $T_{d}$ for successfully received packets as a function of different number of nodes $N=10,30$ and $m_{b}=5,8$ with given $m_{0}=3, m=4, n=3$ and a fixed length of packet $L=70$ bytes.

and a probability $P_{c}$ to initialize the backoff procedure until the maximum retransmission limit is reached after $C_{t}(Z)$. Let $\mathcal{S}_{j}, \mathcal{C}_{j}$ be the event of successful transmissions and discarded packet due to channel access failure after $j$ collisions, respectively. Denote with $\mathcal{F}$ the event of discarded packets due to retry limits and $\mathcal{U}_{t}$ all the possible events $\mathcal{S}_{j}, \mathcal{C}_{j}, \mathcal{F}$. By considering the normalization with $\operatorname{Pr}\left(\mathcal{U}_{t}\right)$ in (9), the probabilities $\operatorname{Pr}\left(\mathcal{S}_{j} \mid \mathcal{U}_{t}\right), \operatorname{Pr}\left(\mathcal{C}_{j} \mid \mathcal{U}_{t}\right), \operatorname{Pr}\left(\mathcal{F} \mid \mathcal{U}_{t}\right)$ are given by Eqs. (6), (7), (8), respectively. Since a packet is transmitted after successful channel access during two CCAs, the probability for successful channel access is $1-(\alpha+$ $(1-\alpha) \beta)^{m+1}$. Note that $H_{m+1}(Z), G_{m+1}(Z)$ consider the random backoff process and sensing delay for discarded packets due to channel sensing fails, respectively. We derive the generalized state transition diagram $B(Z)$ for the packet transmission process as shown in Fig. 2. $B(Z)$ is simply the signal transfer function from the start state to the end state as a function of $P_{c}, \alpha, \beta, m, n$ and $Z . B(Z)$ is given by the sum of three terms: the first one is the successful packet transmission, the second and third terms consider the time period of discarded packet due to the channel access failure and retry limits, respectively. Then, by including Eqs. (3), (4) and (6), the PGF $B(Z)$ given in (5) follows.

Proposition 2: Let $D(Z)$ be the PGF of the packet delay $T_{d}$ for successfully received packet. Then,

$$
\begin{aligned}
D(Z)= & S_{t}(Z) \sum_{j=0}^{n} \operatorname{Pr}\left(\mathcal{A}_{j} \mid \mathcal{A}_{t}\right) C_{t}(Z)^{j} \\
& \times\left(S_{c}(Z)^{2} \sum_{i=0}^{m} \frac{G_{i}(Z) H_{i}(Z)}{\sum_{k=0}^{m} C_{\alpha \beta}(k)}\right)^{j+1},
\end{aligned}
$$

where

$$
\operatorname{Pr}\left(\mathcal{A}_{j} \mid \mathcal{A}_{t}\right)=\frac{\left(1-P_{c}\left(1-x^{m+1}\right)\right)\left(P_{c}\left(1-x^{m+1}\right)\right)^{j}}{1-\left(P_{c}\left(1-x^{m+1}\right)\right)^{n+1}} .
$$

Proof: Let $\mathcal{A}_{j}$ be the event of a successful transmission at time $j+1$ after $j$ collisions and $\mathcal{A}_{t}$ be event of successful transmission within the total attempts $n$. The probability of $\mathcal{A}_{j}$ given that a node transmits packet successfully is obtained as (11). From Eq. (5), the PGF of packet delay for successfully received packet follows after simple passages.
The results provided us by the previous propositions are used to derive the probability distribution function of $T_{s}$ and $T_{d}$, as explained in Eqs. (1) and (2).

Since $D(Z)$ is a smooth function of $Z$, it can be written as power series

$$
D(Z)=\sum_{i=0}^{\infty} \operatorname{Pr}\left(T_{d}=i\right) Z^{i},
$$

and we can obtain the mean value and variance of the packet delay $T_{d}$ from $D(Z)$. The same argument applies for the service time $T_{s}$, which is derived by $B(Z)$.

\section{Simulations and Numerical Results}

Here we first present extensive Monte Carlo simulations to validate our theoretical results based on the specifications of the IEEE 802.15.4 [2]. We also investigate the effects of traffic load, number of nodes, length of the packet and the protocol parameters $\left(m_{0}, m_{b}, m, n\right)$ in terms of delay $T_{d}$. Furthermore, we show the effect of protocol parameters on the stability of control systems.

\section{A. Validation of Delay Distribution}

We validate the probability distribution for the delay $T_{d}$ of successfully received packets. We show that such a probability distribution matches well the simulation results, which means that we can compute the average and variance of the delay $T_{d}$ accurately, as discussed in Section III.

Fig. 3 shows the probability distribution of the delay $T_{d}$ as a function of different number of nodes $N=10,30$ and $m_{b}=5,8$ with given $m_{0}=3, m=4, n=3$ and a fixed length of packet $L=70$ bytes. We observe that the analytical probability distribution predicts well the simulation results. The low traffic $q=0.9$ gives a distribution similar to a deterministic one. This is due to that the collision probability is lower as traffic decreases and most of packets are successfully transmitted at the first backoff stage without channel sensing fails or retransmissions. The saturated traffic $q=0$ results in a heavy tail of the delay distribution due to the high interference. Figs. 3(a), 3(b) show the effect of the number of nodes in terms of the delay. It is interesting to observe that as the number of nodes increases the side lobe increases. This is due to that as the traffic and number of nodes increase, the busy channel probability $\alpha, \beta$ and collision probability $P_{c}$ are also increasing. 


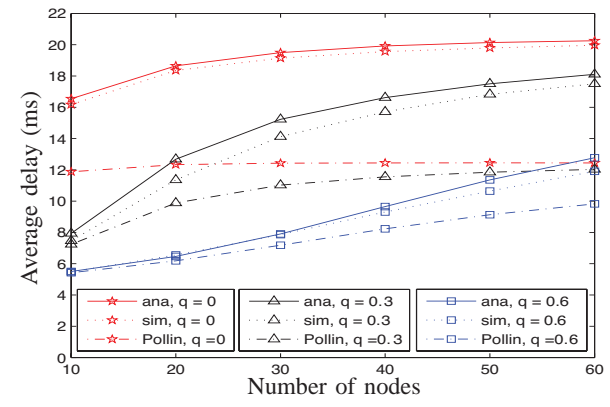

Fig. 4. Average of the delay $T_{d}$ as a function of traffic load $q=0,0.3,0.6$ with a given length of packet $L=70$ bytes and default parameters $\left(m_{0}=3, m_{b}=5, m=4, n=3\right)$ compared with Pollin's Markov chain model [14].

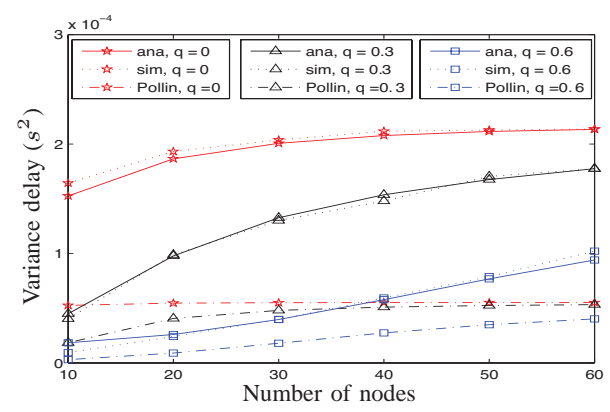

Fig. 5. Variance of the delay $T_{d}$ as a function of traffic load $q=0,0.3,0.6$ with a given length of packet $L=70$ bytes and default parameters $\left(m_{0}=\right.$ $3, m_{b}=5, m=4, n=3$ ) compared to Pollin's Markov chain model [14].

By comparing Figs. 3(a), 3(b) to 3(c), 3(d), we observe that $m_{b}=8$ gives a longer tail than $m_{b}=5$. The probability of delay larger than $50 \mathrm{~ms}$ is almost zero for $m_{b}=5$ while the probability of delay larger than $50 \mathrm{~ms}$ is still nonzero for $m_{b}=8$. Hence, the tail for $m_{b}=8$ is much longer than the distribution of $m_{b}=5$. The random backoff time increases for increasing $m_{b}$. As the traffic, number of nodes and $m_{b}$ increase, the probability distribution has a longer tail. Remark that the probability distribution of the delay is significantly different from the existing network models used for NCS design such as a Poisson distribution [5] and exponential distribution [17].

\section{B. Effect of Network and Protocol Parameters}

We evaluate the average and variance of delay given in Eqs. (1) and (2), respectively, and analytical model [14] for different number of nodes $N=10, \ldots, 60$, as a function of traffic loads $q=0,0.3,0.6$, whereas a fixed length $L$ of the packet and default protocol parameters $\left(m_{0}=3, m_{b}=\right.$ $5, m=4, n=3$ ) are used.

Figs. 4 and 5 report the effect of the number of nodes on the average and variance of the delay $T_{d}$ as a function of the traffic load. In the figure, note that "Pollin" refers to the analytical expression which is derived from the Markov model in [14]. The analytical models of Eqs. (1) and (2) follow well the simulation results, while Pollin's model has weak matching since it does not consider retransmissions. Such a weak matching increases as the number of nodes

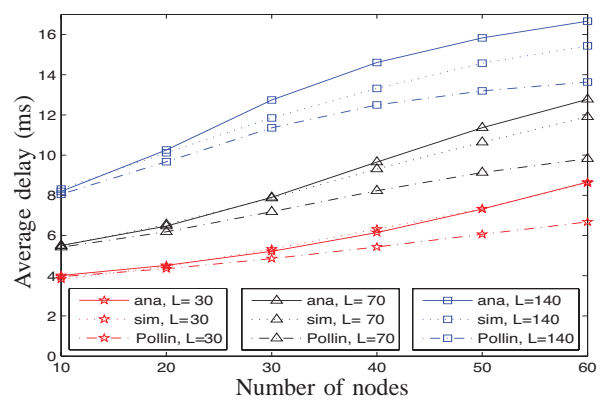

Fig. 6. Average of the delay $T_{d}$ as a function of the packet sizes $L=$ $30,70,140$ bytes with given traffic load $q=0.6$ and default parameters $\left(m_{0}=3, m_{b}=5, m=4, n=3\right)$ compared to Pollin's Markov chain model [14].

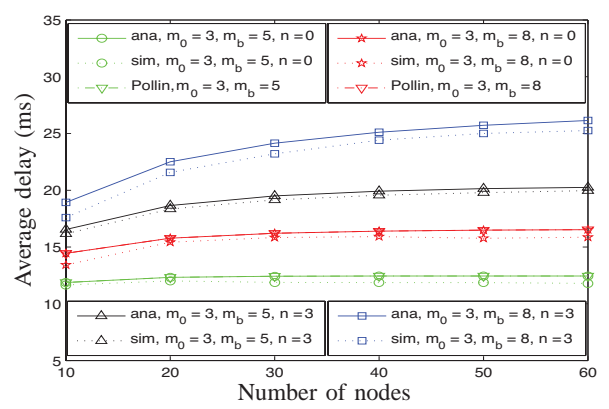

Fig. 7. Average of the delay $T_{d}$ as a function of different CSMA/CA parameters $m_{0}=3, m_{b}=5,8, n=0,3, m=4$ with given traffic load $q=0$ and packet size $L=70$ bytes compared to Pollin's Markov chain model [14].

increases due to higher busy channel and collision probability for the limited number of retransmissions. We observe that the average delay is linearly increasing when the traffic load is low $q=0.6$. Recall that the traffic $q=0$ models the saturated traffic. Note that the busy channel probabilities $\alpha, \beta$ and collision probability $P_{c}$ are mainly dependent on the number of nodes at saturated traffic model $q=0$. The average and variance of delay are saturated for $N \geq 50$ due to high busy channel probability and collision probability. In addition, we observe a strong dependence between average delay and variance of delay.

Fig. 6 illustrates the average delay as achieved by the analytical model and simulations as a function of the packet size $L=30,70,140$ bytes with unsaturated traffic load $q=$ 0.6 and default parameters. The good matching between the analytical model and simulation results is evident. Observe that the average delay is linearly increasing as the number of nodes increases when the length of packet is small, and that the busy channel and collision probabilities decrease as the length of packet decreases. The accuracy of the model slightly decreases when the length of packets increases, due to the assumption of the independent channel access probability in Section II. However, for control applications, the packet size is typically smaller than 20 bytes [1].

Fig. 7 shows the average of the delay $T_{d}$ as a function of different protocol parameters $\left(m_{0}=3, m_{b}=5,8, m=\right.$ $4, n=0,3)$ for saturated traffic model $q=0$ and a 
given length of packet $L=70$ bytes. The analytical model matches well the simulation results. As we have mentioned in Section IV-A, the average delay increases as the parameter $m_{b}$ increases. This is due to that as the parameter $m_{b}$ increases the maximum random backoff time is also increasing. Observe that the average delay is strongly dependent on retry limits $n=0,3$, even if we consider saturated traffic load.

\section{Effect of the Network Parameter on Control Systems}

In this section, we illustrate the effect of the protocol parameter on the stability of a control system. We consider a Linear Time Invariant (LTI) system, where each sensor transmits measurements of a plant to a controller over a WPAN. We consider the state-space model given in [4]:

$$
\begin{aligned}
& \dot{\mathbf{x}}(t)=\left[\begin{array}{ll}
0 & 5 \\
0 & 0
\end{array}\right] \mathbf{x}(t)+\left[\begin{array}{l}
0 \\
1
\end{array}\right] \mathbf{u}(t) \\
& \mathbf{y}(\mathbf{t})=\left[\begin{array}{ll}
1 & 0
\end{array}\right] \mathbf{x}(t)
\end{aligned}
$$

where $\mathbf{u}(t)=-\left[\begin{array}{ll}25 & 10\end{array}\right] \mathbf{x}(t)$ is the feedback state control decision. The delay of such a transmission is modeled statistically by the results given in the previous sections. We assume that all packets are successfully transmitted so to observe the pure effect of packet delay distribution on the stability of the control system.

In Fig. 8, we compare the step response of the packet delay with different protocol parameters $m_{0}=3,7,8, m_{b}=8$, $n=0, m=4$ with respect to ideal step response when the delay is ignored at a fixed sampling period $h=0.02 \mathrm{~s}$. Recall that the packet delay distribution is heavily affected by the parameters of CSMA/CA mechanism as we observed in Section IV-A and IV-B. The packet delay causes the closedloop plant to be underdamped, resulting in larger overshoot in the step response. Observe that as the CSMA/CA parameter $m_{0}$ increases, the settling time of control system increases. Moreover, the system is unstable when the parameter $m_{0}=8$ due to a longer packet delay and higher delay variance. The heavy tail of packet delay distribution degrades the stability of LTI systems. In [15], we show that the packet loss probability decreases as the parameter $m_{0}$ increases. However, there is a tradeoff between the packet loss probability and the delay jitter in WPANs. Therefore, the selection of the parameter plays a fundamental role for the stability.

\section{Conclusions}

In this paper, we characterized the probability distribution functions of the service time and the delay to receive packet successfully for the CSMA/CA mechanism of WPANs. The explicit expression of the delay distribution is a function of the traffic load, number of nodes, length of data packets, and protocol parameters (macMinBE, macMaxBE, macMaxCSMABackoffs, macMaxFrameRetries). Monte Carlo simulations validated the analysis. If the traffic load is very low, then the average delay is linearly increasing with the number of nodes. The delay distribution is significantly different from commonly used delay models of NCSs. Furthermore, the protocol parameters affect the delay distribution and the performance of a state feedback control system.

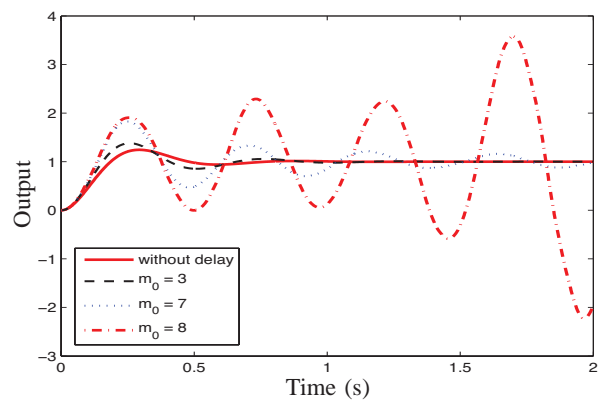

Fig. 8. Effect of the packet delay distribution on a state feedback control system over a WPAN. The sampling period is $h=0.02 \mathrm{~s}$, the packet size is $L=30$ bytes with CSMA/CA parameters $m_{0}=3,7,8, m_{b}=8, n=0$, and $m=4$ and a number of nodes $N=10$.

\section{REFERENCES}

[1] A. Willig, "Recent and emerging topics in wireless industrial communication," IEEE Transactions on Industrial Informatics, vol. 4, no. 2, pp. 102-124, 2008.

[2] IEEE 802.15.4: Wireless Medium Access Control (MAC) and Physical Layer (PHY) Specifications for Low-Rate Wireless Personal Area Networks (WPANs), IEEE, 2006. [Online]. Available: http://www.ieee802.org/15

[3] J. Nilsson, B. Bernhardsson, and B. Wittenmark, "Stochastic analysis and control of real-time systems with random time delays," Automatica, vol. 34, no. 1, pp. 57-64, 1998.

[4] W. Zhang and M. S. Branicky and S. M. Phillips, "Stability of Networked Control Systems," IEEE Control Systems Magazine, vol. 21, pp. 84-99, 2001.

[5] G. Walsh, H. Ye, and L. Bushnell, "Stability analysis of networked control systems," IEEE Transactions on Control Systems Technology, vol. 10, no. 3, pp. $438-446,2002$.

[6] J. P. Hespanha, P. Naghshtabrizi, and Y. Xu, "A survey of recent results in networked control systems," Proceedings of the IEEE, vol. 95, no. 1, pp. 138-162, 2007.

[7] IEEE 802.11 Wireless LAN Medium Access Control (MAC) and Physical Layer (PHY) Specifications, IEEE, 1999. [Online]. Available: http://www.ieee802.org/11

[8] L. Montestruque and P. Antsaklis, "Stability of model-based networked control systems with time-varying transmission times," IEEE Transactions on Automatic Control, vol. 49, no. 9, pp. 1562 - 1572, 2004.

[9] J. Nilsson and B. Bernhardsson, "LQG control over a Markov communication network," in IEEE CDC, 1997.

[10] M. Donkers, W. Heemels, D. Bernardini, A. Bemporad, and V. Shneer, "Stability analysis of stochastic networked control systems," in ACC, 2010.

[11] J. Zheng and M. L. Lee, "A comprehensive performance study of IEEE 802.15.4," in IEEE Press Book, 2004.

[12] G. Bianchi, "Performance analysis of the IEEE 802.11 distributed cordination function," in IEEE Journal on Selected Areas in Communications, vol. 18 , no. 3, 2000, pp. 535 - 547.

[13] O. Tickioo and B. Sikdar, "Queueing analysis and delay mitigation in IEEE 802.11 random access MAC based wireless networks," in Proc. of IEEE INFOCOM, 2004.

[14] S. Pollin, M. Ergen, S. C. Ergen, B. Bougard, F. Catthoor, A. Bahai, and P. Varaiya, "Performance analysis of slotted carrier sense IEEE 802.15.4 acknowledged uplink transmissions," in Proc. of IEEE WCNC, 2008.

[15] P. Park, P. D. Marco, P. Soldati, C. Fischione, and K. H. Johansson, "A generalized markov chain model for effective analysis of slotted IEEE 802.15.4," in Proc. of IEEE MASS, 2009.

[16] P. Park, J. Araujo, and K. H. Johansson, "Wireless networked control system co-design," in IEEE ICNSC, 2011.

[17] M. Tabbara and D. Nesic, "Inputcoutput stability of networked control systems with stochastic protocols and channels," IEEE Transactions on Automatic Control, vol. 53, no. 5, pp. 1160 -1175, 2008. 